\title{
On Some Properties of Cowen-Douglas Class of Operators
}

\author{
Parastoo Heiatian Naeini and Bahmann Yousefi \\ Department of Mathematics, Payame Noor University, P.O. Box 19395-3697, Tehran, Iran \\ Correspondence should be addressed to Bahmann Yousefi; b_yousefi@pnu.ac.ir \\ Received 9 September 2017; Accepted 29 November 2017; Published 1 February 2018 \\ Academic Editor: Kehe Zhu
}

Copyright (C) 2018 Parastoo Heiatian Naeini and Bahmann Yousefi. This is an open access article distributed under the Creative Commons Attribution License, which permits unrestricted use, distribution, and reproduction in any medium, provided the original work is properly cited.

\begin{abstract}
We will consider multiplication operators on a Hilbert space of analytic functions on a domain $\Omega \subset \mathbb{C}$. For a bounded analytic function $\varphi$ on $\Omega$, we will give necessary and sufficient conditions under which the complement of the essential spectrum of $M_{\varphi}$ in $\varphi(\Omega)$ becomes nonempty and this gives conditions for the adjoint of the multiplication operator $M_{\varphi}$ belongs to the Cowen-Douglas class of operators. Also, we characterize the structure of the essential spectrum of a multiplication operator and we determine the commutants of certain multiplication operators. Finally, we investigate the reflexivity of a Cowen-Douglas class operator.
\end{abstract}

\section{Introduction}

In this section we include some preparatory material which will be needed later.

For a positive integer $n$ and a domain $U \subset \mathbb{C}$, the CowenDouglas class $B_{n}(U)$ consists of bounded linear operators $T$ on any fixed separable infinite dimensional Hilbert space $X$ with the following properties:

(a) $U$ is a subset of the spectrum of $T$.

(b) $\operatorname{Ran}(\lambda-T)=X$ for every $\lambda \in U$.

(c) $\operatorname{dim}[\operatorname{ker}(\lambda-T)]=n$ for every $\lambda \in U$.

(d) $\operatorname{Span}\{\operatorname{ker}(\lambda-T): \lambda \in U\}=X$.

Here Span denotes the closed linear span of a collection of sets in $X$. The classes $B_{n}(U)$ were introduced by CowenDouglas (see [1]), and each element of $B_{n}(U)$ is called a Cowen-Douglas class operator. By $B_{n}$, we mean $B_{n}(U)$ for some complex domain $U$. For the study of Cowen-Douglas classes $B_{n}$, we mention [1-7].

Recall that a bounded linear operator $A$ on a Hilbert space is a Fredholm operator if and only if $\operatorname{ran} A$ is closed and both ker $A$ and ker $A^{*}$ are finite dimensional. We use $\sigma(A)$ and $\sigma_{e}(A)$ to denote, respectively, the spectrum of $A$ and the essential spectrum of $A$.

Now let $\mathscr{H}$ be a separable Hilbert space and let $\mathscr{B}(\mathscr{H})$ denote the algebra of all bounded linear operators on $\mathscr{H}$. Recall that if $A \in \mathscr{B}(\mathscr{H})$, then $\operatorname{Lat}(A)$ is by definition the lattice of all invariant subspaces of $A$, and $\operatorname{AlgLat}(A)$ is the algebra of all operators $B$ in $\mathscr{B}(\mathscr{H})$ such that $\operatorname{Lat}(A) \subset \operatorname{Lat}(B)$. An operator $A$ in $\mathscr{B}(\mathscr{H})$ is said to be reflexive if $\operatorname{AlgLat}(A)=$ $W(A)$, where $W(A)$ is the smallest subalgebra of $\mathscr{B}(\mathscr{H})$ that contains $A$ and the identity $I$ and is closed in the weak operator topology.

Also, if $\mathscr{H}$ is a Hilbert space of functions analytic on a plane domain $\Omega$, a complex-valued function $\varphi$ on $\Omega$ for which $\varphi f \in \mathscr{H}$ for every $f \in \mathscr{H}$ is called a multiplier of $\mathscr{H}$ and the multiplier $\varphi$ on $\mathscr{H}$ determines a multiplication operator $M_{\varphi}$ on $\mathscr{H}$ by $M_{\varphi} f=\varphi f, f \in \mathscr{H}$. The set of all multipliers of $\mathscr{H}$ is denoted by $M(\mathscr{H})$. Clearly $M(\mathscr{H}) \subset H^{\infty}(\Omega)$, where $H^{\infty}(\Omega)$ is the space of all bounded analytic function on $\Omega$. In fact $\|\varphi\|_{\infty} \leq\left\|M_{\varphi}\right\|$ (see [8]).

Let $\mathscr{H}$ be a Hilbert space of functions analytic on a domain $\Omega \subset \mathbb{C}$ satisfying the following axioms:

Axiom 1. For every point $\omega \in \Omega$, the functional of point evaluation at $\omega$, is a nonzero bounded linear functional on $\mathscr{H}$.

Axiom 2. Every function $\varphi \in H^{\infty}(\Omega)$ is a multiplier of $\mathscr{H}$.

Axiom 3. If $f \in \mathscr{H}$ and $f(\lambda)=0$, then there is a function $g \in \mathscr{H}$ such that $(z-\lambda) g=f$.

A space $\mathscr{H}$ satisfying the above conditions is called Hilbert space of analytic functions on $\Omega$ (see $[3,9]$ ). The Hardy and 
Bergman spaces are examples for Hilbert spaces of analytic functions on the open unit disk.

Note that, by Axiom 1, there exists a reproducing kernel $k_{w} \in \mathscr{H}$ such that $f(\omega)=\left\langle f, k_{\omega}\right\rangle$ for all $f \in \mathscr{H}$. Also, by using Axiom 2 and the closed-graph theorem, the operator of multiplication by $\varphi, M_{\varphi}$, is a bounded linear operator on $\mathscr{H}$. So Axiom 2 says that $M(\mathscr{H})=H^{\infty}(\Omega)$. If $M_{z}$ is polynomially bounded on $\mathscr{H}$ and $\Omega$ is the open unit disk, then $M(\mathscr{H})=$ $H^{\infty}(\Omega)$ (see [9, Theorem 1]). In the rest of the paper we assume that $\mathscr{H}$ is a Hilbert space of analytic function on a bounded plane domain $\Omega$.

In this paper, we want to study some properties of operators in $B_{n}$. We see that complement of the essential spectrum of a multiplication operator $M_{\varphi}$ is nonempty if and only if the adjoint of $M_{\varphi}$ belongs to some $B_{n}$. Also, we investigate the intertwining multiplication operators and reflexivity of the multiplication operator on $B_{n}$. For some other source on these topics one can see [10-16].

\section{Multiplication Operators with Adjoint in $B_{n}$ and Its Spectra}

Recall that if $T$ is a Cowen-Douglas class operator, then it should be $\sigma(T) \backslash \sigma_{e}(T) \neq \emptyset$. For $\varphi \in H^{\infty}(\Omega)$, we would like to give some necessary and sufficient conditions so that $\sigma\left(M_{\varphi}\right) \backslash \sigma_{e}\left(M_{\varphi}\right)$ becomes a nonempty open set. This implies a sufficient condition for the adjoint of the multiplication operator $M_{\varphi}$ to be a Cowen-Douglas class operator.

Theorem 1. Let $\varphi$ be a nonconstant function in $H^{\infty}(\Omega)$, $\sigma\left(M_{\varphi}\right) \backslash \sigma_{e}\left(M_{\varphi}\right) \neq \emptyset$, and $k_{z} /\left\|k_{z}\right\| \rightarrow 0$ weakly as $\operatorname{dist}(z, \partial \Omega) \rightarrow 0$. Then there exist a domain $V \subset \varphi(\Omega)$ and a positive integer $n$ such that $\Omega \cap \varphi^{-1}(\lambda)$ consists of $n$ points (counting multiplicity) for every $\lambda \in V$.

Proof. First note that if $\lambda \in \varphi(\Omega)$, then $\lambda=\varphi(\omega)$ for some $\omega \in \Omega$. But by Axiom 1, the functional of evaluation at $\omega$ is a bounded point evaluation; thus the reproducing kernel $k_{\omega}$ is defined and we have

$$
M_{\varphi}^{*} k_{\omega}=\overline{\varphi(\omega)} k_{\omega} .
$$

Thus $\lambda \in \sigma\left(M_{\varphi}\right)$ and clearly $\overline{\varphi(\Omega)} \subset \sigma\left(M_{\varphi}\right)$. Now let $\lambda \notin$ $\overline{\varphi(\Omega)}$. Then $\varphi-\lambda$ is an invertible element of $H^{\infty}(\Omega)$. But by Axiom 2, we have $M(\mathscr{H})=H^{\infty}(\Omega)$; thus $M_{\varphi-\lambda}$ is invertible. This implies that $\sigma\left(M_{\varphi}\right) \subset \overline{\varphi(\Omega)}$; thus indeed $\sigma\left(M_{\varphi}\right)=\overline{\varphi(\Omega)}$. Now we prove that

$$
\sigma\left(M_{\varphi}\right) \backslash \sigma_{e}\left(M_{\varphi}\right)=\varphi(\Omega) \backslash \sigma_{e}\left(M_{\varphi}\right)
$$

For this it is sufficient to show that $\partial \varphi(\Omega) \subset \sigma_{e}\left(M_{\varphi}\right)$. Let $\lambda \notin$ $\sigma_{e}\left(M_{\varphi}\right)$. If $\lambda \in \partial \varphi(\Omega)$, then there exists a sequence $\left\{z_{n}\right\}_{n} \subset \Omega$ such that $\varphi\left(z_{n}\right) \rightarrow \lambda$. By passing to a subsequence if necessary, we may assume that $\left\{z_{n}\right\}_{n}$ converges to a point in $\partial \Omega$ and so by our assumptions $k_{z_{n}} /\left\|k_{z_{n}}\right\| \rightarrow 0$ weakly. On the other hand we have

$$
\left(M_{\varphi}-\lambda\right)^{*}\left(\frac{k_{z_{n}}}{\left\|k_{z_{n}}\right\|}\right)=\frac{\left(\overline{\varphi\left(z_{n}\right)}-\bar{\lambda}\right) k_{z_{n}}}{\left\|k_{z_{n}}\right\|}
$$

for all $n \in \mathbb{N}$. So we get

$$
\left\|\left(M_{\varphi}-\lambda\right)^{*}\left(\frac{k_{z_{n}}}{\left\|k_{z_{n}}\right\|}\right)\right\| \longrightarrow 0
$$

which contradicts the fact that $\left(M_{\varphi}-\lambda\right)^{*}$ is Fredholm. Thus we have

$$
\sigma\left(M_{\varphi}\right) \backslash \sigma_{e}\left(M_{\varphi}\right)=\varphi(\Omega) \backslash \sigma_{e}\left(M_{\varphi}\right)
$$

Now, let $V$ be a connected component of the open set $\varphi(\Omega) \backslash$ $\sigma_{e}\left(M_{\varphi}\right)$. Since $V \cap \sigma_{e}\left(M_{\varphi}\right)=\emptyset$, thus $M_{\varphi}-\lambda$ is Fredholm for every $\lambda$ in $V$. Also, note that if $\left(M_{\varphi}-\lambda\right)^{\varphi} f=0$, then $f=0$ on $\Omega \backslash(\varphi-\lambda)^{-1}\{0\}$ which is open. Hence $f \equiv 0$ and so $M_{\varphi}-\lambda$ is injective. Thus

$$
\operatorname{index}\left(M_{\varphi}-\lambda\right)^{*}=\operatorname{dim}\left[\operatorname{ker}\left(M_{\varphi}-\lambda\right)^{*}\right]
$$

for all $\lambda$ in $V$. But the index function is continuous from the set of semi-Fredholm operators into $\mathbb{Z} \cup\{ \pm \infty\}$ with discrete topology; thus, index $\left(M_{\varphi}-\lambda\right)^{*}$ is constant for all $\lambda$ in $V$. Put

$$
\operatorname{dim}\left[\operatorname{ker}\left(M_{\varphi}-\lambda\right)^{*}\right]=n
$$

If $z \in V$, then $\lambda=\varphi\left(\lambda_{0}\right)$ for some $\lambda_{0} \in \Omega$ and so $M_{\varphi}^{*} k_{\lambda_{0}}=$ $\bar{\lambda} k_{\lambda_{0}}$. Thus $k_{\lambda_{0}} \in \operatorname{ker}\left(M_{\varphi}-\lambda\right)^{*}$. Since a finite subset of points $\omega$ in $\Omega$ yields a linearly set independent set of functions $k_{\omega}$ in $\mathscr{H}$, thus $\Omega \cap \varphi^{-1}(\lambda)$ consist of at most $n$ points for all $\lambda$ in $V$. So for each fixed $\lambda \in V$, there exist $\lambda_{1}, \lambda_{2}, \ldots, \lambda_{m}$ in $\Omega$ and $n_{1}, n_{2}, \ldots, n_{m}$ in $\mathbb{N}$ such that $m \leq n$ and for all $z \in \Omega$ we have

$$
\varphi(z)-\lambda=\psi(z)\left(z-\lambda_{1}\right)^{n_{1}}\left(z-\lambda_{2}\right)^{n_{2}} \cdots\left(z-\lambda_{m}\right)^{n_{m}},
$$

where $\psi$ belongs to $H^{\infty}(\Omega)$ and is nonvanishing on $\Omega$. Now by a method used in the proof of [3, Proposition 3.1] we show that the function $\psi$ is also bounded below on $\Omega$. For this choose $r>0$ such that $\overline{B(\lambda, r)}$ is contained in $V$. Put $K=$ $\varphi^{-1}(\overline{B(\lambda, r))}$, and thus $K$ is a compact subset of $\Omega$ and so it has a positive distance $\delta$ to $\partial \Omega$. Now if $\psi$ is not bounded below on $\Omega$, then there exists a sequence $\left\{z_{i}\right\}$ in $\Omega-\left\{\lambda_{1}, \lambda_{2}, \ldots, \lambda_{n}\right\}$ such that $\psi\left(z_{i}\right) \rightarrow 0$ as $i \rightarrow \infty$. Since $\psi$ is nonvanishing on $\Omega$ implies that $\varphi\left(z_{i}\right) \rightarrow \lambda$, so there exists a positive integer $N$ such that $\varphi\left(z_{i}\right) \in \overline{B(\lambda, r)}$ for all $i>N$. Hence $z_{i} \in K$ for all $i>N$ that is contradiction to $z_{i} \rightarrow \partial \Omega$. Thus the function $\psi$ is indeed bounded below on $\Omega$. Now since $\psi$ is bounded below and bounded above on $\Omega$ it is an invertible element of $H^{\infty}(\Omega)$ and so the operator $M_{\psi}$ is invertible on $\mathscr{H}$ because $M(\mathscr{H})=H^{\infty}(\Omega)$. Thus index $\left(M_{\psi}\right)=0$. Note that since

$$
\begin{aligned}
M_{\varphi} & -\lambda \\
& =M_{\psi}\left(M_{z}-\lambda_{1}\right)^{n_{1}}\left(M_{z}-\lambda_{2}\right)^{n_{2}} \cdots\left(M_{z}-\lambda_{m}\right)^{n_{m}},
\end{aligned}
$$

we get

$$
\operatorname{index}\left(M_{\varphi}-\lambda\right)=\sum_{j=1}^{m} n_{j}\left(\operatorname{index}\left(M_{z}-\lambda_{j}\right)\right) \text {. }
$$


But $M_{\varphi}-\lambda$ is injective for all $\lambda \in V$; thus

$$
\operatorname{index}\left(M_{\varphi}-\lambda\right)=-\operatorname{dim}\left[\operatorname{ker}\left(M_{\varphi}-\lambda\right)^{*}\right]=-n \text {. }
$$

Clearly, $M_{z}-\lambda_{j}$ is injective; thus

$$
\text { index }\left(M_{z}-\lambda_{j}\right)=-\operatorname{dim}\left[\operatorname{ker}\left(M_{z}-\lambda_{j}\right)^{*}\right]
$$

for $j=1, \ldots, m$. Note that, by Axiom 3 on $\mathscr{H}, \operatorname{ker}\left(M_{z}-\lambda_{j}\right)^{*}$ is one-dimensional (see [17]); thus $\sum_{j=1}^{m} n_{j}=n$ and therefore $\Omega \cap \varphi^{-1}(\lambda)$ consists of exactly $n$ points (counting multiplicity) for every $\lambda \in V$ and now the proof is complete.

From the proof of Theorem 1, we can conclude the following result.

Corollary 2. Let $\varphi$ be a nonconstant function in $H^{\infty}(\Omega)$ and $k_{z} /\left\|k_{z}\right\| \rightarrow 0$ weakly as $\operatorname{dist}(z, \partial \Omega) \rightarrow 0$. Then $\partial \varphi(\Omega) \subset$ $\sigma_{e}\left(M_{\varphi}\right)$.

Note that, by Axiom 3, for every $\lambda \in \Omega$ the operator $M_{z-\lambda}$ is bounded below on $\mathscr{H}$ and also the space $\mathscr{H} \ominus(z-$ $\lambda) \mathscr{H}$ is one-dimensional (see [3]). So the Hilbert space under consideration, $\mathscr{H}$, satisfies the conditions assumed by Zhu in [7].

The following result was stated by Zhu in [7, Proposition 5.2], but its proof is left to readers. For this reason we sketch a proof of this proposition and although our proof might seem more straightforward than the one stated by Zhu, we emphasise that our main idea is given from [7].

Proposition 3. Suppose $\varphi \in H^{\infty}(\Omega)$ and $V$ is a domain contained in $\varphi(\Omega)$. If there exists a positive integer $n$ such that $\Omega \cap \varphi^{-1}(\lambda)$ consists of $n$ points (counting multiplicity) for every $\lambda \in V$, then the adjoint of the operator $M_{\varphi}: \mathscr{H} \rightarrow \mathscr{H}$ belongs to the Cowen-Douglas class $B_{n}(U)$, where $U=\{\bar{z}: z \in V\}$.

Proof. Let $\lambda=\varphi(\omega) \in V$. Then there exist an invertible function $\psi \in H^{\infty}(\Omega)$ and $z_{1}, z_{2}, \ldots, z_{m} \in \Omega \cap \varphi^{-1}(\lambda)$ such that

$$
\begin{aligned}
& \left(M_{\varphi}-\lambda\right)^{*} \\
& \quad=M_{\psi}^{*}\left(M_{z}-z_{1}\right)^{*^{k_{1}}}\left(M_{z}-z_{2}\right)^{*^{k_{2}}} \cdots\left(M_{z}-z_{m}\right)^{*^{k_{m}}},
\end{aligned}
$$

where $\sum_{i=1}^{m} k_{i}=n$. Axiom 3 implies that for all $i=1, \ldots$, $m,\left(M_{z}-z_{i}\right)^{*}$ is onto (see [17]); thus for all $\lambda \in V,\left(M_{\varphi}-\lambda\right)^{*}$ is onto since $M_{\psi}$ is invertible. Also, by Axiom 3, $\operatorname{dim}\left[\operatorname{ker}\left(M_{z}-\right.\right.$ $\left.\left.z_{i}\right)^{*}\right]=1$ for $i=1, \ldots, m$ and so

$$
\begin{aligned}
& \operatorname{dim}\left[\operatorname{ker}\left(M_{\varphi}-\lambda\right)^{*}\right]=\sum_{i=1}^{m} k_{i} \operatorname{dim}\left[\operatorname{ker}\left(M_{z}-z_{i}\right)^{*}\right] \\
& =\sum_{i=1}^{m} k_{i}=n .
\end{aligned}
$$

Finally, we note that

$$
\begin{aligned}
& \operatorname{Span}\left\{k_{\omega}: \omega \in \varphi^{-1}(V)\right\} \\
& \quad \subset \operatorname{Span}\left\{\operatorname{ker}\left(M_{\varphi}-\lambda\right)^{*}: \lambda \in V\right\} .
\end{aligned}
$$

Now, since $\varphi^{-1}(V)$ is open, $\operatorname{Span}\left\{k_{\omega}: \omega \in \varphi^{-1}(V)\right\}=\mathscr{H}$ and so the proof is complete.

Corollary 4. Under the conditions of Theorem 1, there exist a positive integer $n$ and a domain $U$ in the complex plane such that $M_{\varphi}^{*} \in B_{n}(U)$.

Proof. By Theorem 1 and Proposition 3 it is clear.

Now we investigate the converse of Theorem 1.

Corollary 5. Let $\varphi$ be a nonconstant function in $H^{\infty}(\Omega)$. If there exists a domain $V \subset \varphi(\Omega)$ and a positive integer $n$ such that $\Omega \cap \varphi^{-1}(\lambda)$ consists of $n$ points (counting multiplicity) for every $\lambda \in V$; then $V \subset \sigma\left(M_{\varphi}\right) \backslash \sigma_{e}\left(M_{\varphi}\right)$.

Proof. By Proposition 3, the adjoint of the operator $M_{\varphi}$ : $\mathscr{H} \rightarrow \mathscr{H}$ belongs to the Cowen-Douglas class $B_{n}(U)$, where $U=\{\bar{z}: z \in V\}$. Hence for all $\lambda \in U, M_{\varphi}^{*}-\lambda$ is Fredholm and so clearly $V \subset \sigma\left(M_{\varphi}\right) \backslash \sigma_{e}\left(M_{\varphi}\right)$.

Corollary 6. Let $M_{\varphi}^{*} \in B_{n}(U)$ for some positive integer $n$ and a complex domain $U$. If $k_{z} /\left\|k_{z}\right\| \rightarrow 0$ weakly as dist $(z, \partial \Omega) \rightarrow$ 0 , then $\Omega \cap \varphi^{-1}(\lambda)$ consists of $n$ point (counting multiplicity) for every $\lambda \in V$ where $V=\{\bar{z}: z \in U\}$.

Proof. First note that $M_{\varphi}^{*}-\lambda$ is Fredholm for all $\lambda \in U$; thus

$$
V \subset \sigma\left(M_{\varphi}\right) \backslash \sigma_{e}\left(M_{\varphi}\right)=\overline{\varphi(\Omega)} \backslash \sigma_{e}\left(M_{\varphi}\right) .
$$

But by Corollary 2, $\partial \varphi(\Omega) \subset \sigma_{e}\left(M_{\varphi}\right)$; thus, $V \subset \varphi(\Omega)$. Now if $\lambda \in V$, then $\lambda=\varphi(\omega)$ for some $\omega \in \Omega$ and clearly $k_{\omega} \in \operatorname{ker}\left(M_{\varphi}-\lambda\right)^{*}$. Since $\operatorname{dim}\left[\operatorname{ker}\left(M_{\varphi}-\lambda\right)^{*}\right]=n$ and $\mathrm{a}$ finite subset of points $\omega$ in $\Omega$ yields a linearly independent set of functions $k_{\omega}$ in $\mathscr{H}$, thus $\Omega \cap \varphi^{-1}(\lambda)$ consist of at most $\mathrm{n}$ points for all $\lambda \in V$. Now by the same method used in the proof of Theorem 1, we can see that $\Omega \cap \varphi^{-1}(\lambda)$ consists of exactly $n$ points (counting multiplicity) for every $\lambda \in V$.

Example 7. Consider the Hilbert Bergman space $L_{a}^{2}(\mathbb{D})$ where $\mathbb{D}$ is the open unit disc in the complex domain. Then $L_{a}^{2}(\mathbb{D})$ holds in Axioms 1, 2, and 3 (see [17, Theorem 8.5, page 67]). For the Bergman reproducing kernel function, $k_{z}$, clearly we can see that $\left\|k_{z}\right\| \rightarrow \infty$ as $\operatorname{dist}(z, \partial \mathbb{D}) \rightarrow 0$. So if $p$ is a polynomial, then

$$
\left\langle p, \frac{k_{z}}{\left\|k_{z}\right\|}\right\rangle=\frac{p(z)}{\left\|k_{z}\right\|} \longrightarrow 0
$$

as $\operatorname{dist}(z, \partial \mathbb{D}) \rightarrow 0$. But polynomials are dense in $L_{a}^{2}(\mathbb{D})$; thus $k_{z} /\left\|k_{z}\right\| \rightarrow 0$ weakly as $\operatorname{dist}(z, \partial \mathbb{D}) \rightarrow 0$. Now by Theorem 1 and the proof of Corollary 5, we can see that $M_{\varphi}^{*} \in B_{n}(U)$ for some positive integer $n$ and a complex domain $U$ if and only if $\sigma\left(M_{\varphi}\right) \backslash \sigma_{e}\left(M_{\varphi}\right) \neq \emptyset$.

Proposition 8. Let $\varphi$ be a nonconstant function in $H^{\infty}(\Omega)$ and $k_{z} /\left\|k_{z}\right\| \rightarrow 0$ weakly as $\operatorname{dist}(z, \partial \Omega) \rightarrow 0$. Then

$$
\sigma_{e}\left(M_{\varphi}\right)=\cap_{n} \varphi\left(\left\{z \in \Omega: \operatorname{dist}(z, \partial \Omega)<\frac{1}{n}\right\}\right) .
$$


Proof. Let $\lambda \notin \sigma_{e}\left(M_{\varphi}\right)$; then $M_{\varphi}-\lambda$ is Fredholm. Now we show that $\varphi-\lambda$ is bounded away from zero near $\partial \Omega$. By way of contradiction, let $\left\{z_{n}\right\}_{n} \subset \Omega$ be a sequence such that $\varphi\left(z_{n}\right) \rightarrow \lambda$ and $\left\{z_{n}\right\}_{n}$ converges to a point in $\partial \Omega$. Note that by our assumptions $k_{z_{n}} /\left\|k_{z_{n}}\right\| \rightarrow 0$ weakly and

$$
\left(M_{\varphi}-\lambda\right)^{*}\left(\frac{k_{z_{n}}}{\left\|k_{z_{n}}\right\|}\right)=\frac{\left(\overline{\varphi\left(z_{n}\right)}-\bar{\lambda}\right) k_{z_{n}}}{\left\|k_{z_{n}}\right\|}
$$

for all $n \in \mathbb{N}$. So we get

$$
\left\|\left(M_{\varphi}-\lambda\right)^{*}\left(\frac{k_{z_{n}}}{\left\|k_{z_{n}}\right\|}\right)\right\| \longrightarrow 0 .
$$

This is a contradiction because $\left(M_{\varphi}-\lambda\right)^{*}$ is Fredholm. Hence, $\varphi-\lambda$ is bounded away from zero near $\partial \Omega$ and so there exists $m \in \mathbb{N}$ large enough such that

$$
\inf \left\{|\varphi(z)-\lambda|: \operatorname{dist}(z, \partial \Omega)<\frac{1}{m}\right\}>0 .
$$

This implies that

$$
\lambda \notin \cap_{n} \varphi\left(\left\{z \in \Omega: \operatorname{dist}(z, \partial \Omega)<\frac{1}{n}\right\}\right) .
$$

Conversely, if

$$
\lambda \notin \cap_{n} \varphi\left(\left\{z \in \Omega: \operatorname{dist}(z, \partial \Omega)<\frac{1}{n}\right\}\right),
$$

then $\varphi-\lambda$ is bounded away from zero near $\partial \Omega$. Since the zeros of an analytic function are isolated, thus the zeros of $\varphi-\lambda$ are finite. Let $\lambda_{1}, \lambda_{2}, \ldots, \lambda_{j}$ be all zeros (counting multiplicity) of $\varphi-\lambda$ in $\Omega$ such that

$$
\varphi(z)-\lambda=\psi(z)\left(z-\lambda_{1}\right)\left(z-\lambda_{2}\right) \cdots\left(z-\lambda_{j}\right) .
$$

Clearly the function $\psi$ is invertible on $\Omega$ and so $M_{\psi}$ is bounded below. Also, by Axiom 3 on $\mathscr{H}, M_{z}-\lambda$ is Fredholm for all $i=1, \ldots, j$. This implies that $M_{\varphi}-\lambda$ is Fredholm and so $\lambda \notin \sigma_{e}\left(M_{\varphi}\right)$. So the proof is complete.

\section{Intertwining Multiplication Operators}

The following characterization of the commutant $\{T\}^{\prime}$ of $T$ is given in Theorem 3.7 of [2], which is stated for the convenience of the reader. Note that $K$ is the reproducing kernel for a coanalytic functional Hilbert space $\mathscr{K}$ defined in [2].

Theorem 9. If $S$ is in $B_{n}(\Omega)$ and the operator $X$ commutes with $S$, then there exists an analytic function $\Phi: \Omega \rightarrow B_{n}\left(\mathbb{C}^{n}\right)$ such that $X K(\lambda, \cdot)=K(\lambda, \cdot) \Phi(\lambda)$ (all $\lambda \in \Omega$ ) and for every $f \in \mathscr{K}$, $X^{*} f(\cdot)=(\Phi(\cdot))^{*} f(\cdot)$.

In the following let $\Omega$ be such that if $\lambda \in \Omega$ then $-\lambda \in \Omega$. Also we assume that the composition operator $C_{-z}: \mathscr{H} \rightarrow \mathscr{H}$ defined by $C_{-z} f=f(-z)$ is bounded.
Proposition 10. Suppose that $\varphi \in H^{\infty}(\Omega)$ and there exists a domain $V \subset \varphi(\Omega)$ such that $\Omega \cap \varphi^{-1}(\omega)$ is a singleton for every $\omega \in V$. If $\varphi$ is odd, $S M_{\varphi^{2}}=M_{\varphi^{2}} S$ and $S M_{\varphi^{2 n-1}}-M_{\varphi^{2 n-1}} S$ is compact for some natural number $n$; then $S=M_{h}$ for some $h \in H^{\infty}(\Omega)$.

Proof. Note that, by Proposition 3, the adjoint of the operator $M_{\varphi}: \mathscr{H} \rightarrow \mathscr{H}$ belongs to the Cowen-Douglas class $B_{1}(U)$, where $U=\{\bar{z}: z \in V\}$. If $n=1$, all conditions of Theorem 4 in [5] hold and so there exists $h \in H^{\infty}(\Omega)$ such that $S=M_{h}$. For $n>1$, put

$$
T_{1}=S M_{\varphi^{2 n-1}}-M_{\varphi^{2 n-1}} S .
$$

Clearly $T_{1} M_{\varphi}=-M_{\varphi} T_{1}$ and so by Proposition 3 in [5], there exists $h \in H^{\infty}(\Omega)$ such that $T_{1}=M_{h} C_{-z}$. But $M_{h}=M_{h} C_{-z}$ 。 $C_{-z}$ is compact; thus by the Fredholm Alternative Theorem, $h=0$ and so $T_{1}=0$. Hence $S M_{\varphi^{2 n-1}}=M_{\varphi^{2 n-1}} S$. Now we show that $S M_{\varphi^{2 n-3}}=M_{\varphi^{2 n-3}} S$. Put

$$
T_{2}=S M_{\varphi^{2 n-3}}-M_{\varphi^{2 n-3}} S .
$$

And note that $M_{\varphi^{2}} T_{2}=0$. This implies that $T_{2}=0$, since $\varphi$ is analytic and the zeros of $\varphi$ are at most countable. Therefore $S M_{\varphi^{2 n-3}}=M_{\varphi^{2 n-3}} S$. Now if $n=2$, then $S M_{\varphi}=M_{\varphi} S$ and so by Proposition 4.1 in [7] the proof is complete. Else, by continuing this manner, we can conclude that $S M_{\varphi}=M_{\varphi} S$ which implies that $S=M_{\varphi}$ for some $h \in H^{\infty}(\Omega)$.

Proposition 11. Suppose that $\varphi \in H^{\infty}(\Omega)$ and there exists a domain $V \subset \varphi(\Omega)$ such that $\Omega \cap \varphi^{-1}(\omega)$ is a singleton for every $\omega \in V$. If $\varphi$ is odd, $S M_{\varphi^{2}}=M_{\varphi^{2}} S$, and $S M_{\varphi^{2 n-1}}+M_{\varphi^{2 n-1}} S$ is compact for some natural number $n$, then $S=M_{h} C_{-z}$ for some $h \in H^{\infty}(\Omega)$.

Proof. If $n=1$, put

$$
T_{1}=S M_{\varphi}+M_{\varphi} S .
$$

Then $T_{1} M_{\varphi}=M_{\varphi} T_{1}$. Thus $T_{1}=M_{h_{1}}$ for some $h_{1} \in H^{\infty}(\Omega)$. But $M_{h_{1}}$ is compact, hence $h_{1}=0$ and so $T_{1}=0$. This implies that $S M_{\varphi}=-M_{\varphi} S$. Now by Proposition 3 in [5], $S=M_{h} C_{-z}$ for some $h \in H^{\infty}(\Omega)$. If $n>1$, put $T_{2}=S M_{\varphi^{2 n-1}}+M_{\varphi^{2 n-1}} S$. Then, clearly $M_{\varphi} T_{2}=T_{2} M_{\varphi}$ from which we can conclude that $T_{2}=M_{h_{1}}$ for some $h_{1} \in H^{\infty}(\Omega)$. The compactness of $M_{h_{1}}$ implies that $h_{1}=0$ and so $T_{2}=0$.

Thus $S M_{\varphi^{2 n-1}}=-M_{\varphi^{2 n-1}} S$. Put

$$
T_{3}=S M_{\varphi^{2 n-3}}+M_{\varphi^{2 n-3}} S .
$$

Hence $M_{\varphi^{2}} T_{3}=0$ which implies that $T_{3}=0$. Therefore, $S M_{\varphi^{2 n-3}}=-M_{\varphi^{2 n-3}} S$. If $n=2$, then $S M_{\varphi}=-M_{\varphi} S$ and the proof is complete. If $n>2$, by continuing this manner, finally we can see that $S M_{\varphi}=-M_{\varphi} S$ and this completes the proof.

\section{Reflexivity in Cowen-Douglas Class of Operators}

It is shown in [4] that, under sufficient conditions, an operator $T$ in the Cowen-Douglas class $B_{n}(\Omega)$ can be reflexive, where 
$\Omega$ is a special bounded plane domain. In this section we give some sufficient conditions so that the associated canonical model is reflexive. This answers Question 5.6 in [9, p. 98]. Indeed, we investigate the reflexivity of $B_{n}(\Omega)$, when $\Omega$ is an arbitrary bounded domain.

It is well known that every operator in the class $B_{n}(\Omega)$ is unitarily equivalent to the adjoint of the canonical model associated with a generalized Bergman kernel (g.B.k. for brevity) $K$ (see $[2,6]$ ). Actually $K$ is the reproducing kernel for a coanalytic functional Hilbert space $\mathscr{K}_{\mathscr{K}}$ (briefly $\mathscr{K}$ ) on which we can define the operator $T_{\bar{z}}$ of multiplication by $\bar{z}$. The operator $T=T_{\bar{z}}^{*}$ acting on $\mathscr{K}$ is called the canonical model associated with $K$. We know that, for every $\lambda$ in $\Omega$, $T-\lambda$ is onto and

$$
\operatorname{ker}(T-\lambda)=\operatorname{ran} K(\lambda, \cdot)=\left\{K(\lambda, \cdot) \xi: \xi \in \mathbb{C}^{n}\right\}
$$

and $\operatorname{dim} \operatorname{ker}(T-\lambda)=n$.

Recall that a compact subset $F$ of the plane is a spectral set for a bounded operator $A$ if $F$ contains $\sigma(A)$ and $\|f(A)\| \leq$ $\sup _{z \in F}|f(z)|$ for all rational functions $f$ with poles off $F$. Also, an open connected subset $G$ of the plane is called a Carathéodory region if its boundary equals the boundary of the unbounded component of $\mathbb{C}-\bar{G}$.

It is proved in [4] that if $T$ is in $B_{1}(\Omega)$ and $T^{*}$ is an injective unilateral weighted shift, then $T$ is reflexive. Also, it has been shown that if $T$ is in $B_{n}(\Omega)$, where $\Omega$ is a Carathéodory region such that $\sigma(T)=\bar{\Omega}$ is a spectral set for $T$, then $T$ is reflexive (see [4, Theorem 2]). This implies that if $T$ is a contraction in $B_{n}(\mathbb{D})$ where $\mathbb{D}$ is the open unit disk, then $T$ is reflexive. Here we want to investigate the reflexivity of $T$ on $B_{n}(\Omega)$, where $\Omega$ is an arbitrary domain in $\mathbb{C}$.

Theorem 12. If $T$ is in $B_{n}(\Omega)$, where $\Omega \subset \mathbb{C}$ is an arbitrary domain, then there exists a total set $Y$ such that the weak closure of the set $\{p(T) y: p$ is a polynomial, $y \in Y\}$ contains AlgLat $(T)$.

Proof. Let $K$ be a g.B.k. on $\Omega$ and let $X \in \operatorname{AlgLat}(T)$. Then by Theorem 9 and $[4$, Lemma 1$]$, there exists $\psi \in H^{\infty}(\Omega)$ such that $X K(\lambda, \cdot)=\psi(\lambda) K(\lambda, \cdot)$ for all $\lambda$ in $\Omega$. Now let $F=\left\{\lambda_{n}\right\}_{n=1}^{\infty}$ be dense in $\Omega$ and choose $\xi_{i} \in \mathbb{C}^{n}$ such that $K\left(\lambda_{i}, \cdot\right) \xi_{i} \neq 0$ for $i=1,2, \ldots$. Put $\mathscr{K}_{i}=\vee\left\{K\left(\lambda_{i}, \cdot\right) \xi_{i}\right\}$ for $i=1,2, \ldots$. Define

$$
\begin{aligned}
& \mathscr{K}_{\infty}^{\prime}=\mathscr{K}_{1} \oplus \mathscr{K}_{2} \oplus \mathscr{K}_{3} \oplus \cdots=\bigoplus_{i=1}^{\infty} \mathscr{K}_{i}, \\
& T_{\infty}=\left.\left.\left.T\right|_{\mathscr{K}_{1}} \oplus T\right|_{\mathscr{K}_{2}} \oplus T\right|_{\mathscr{K}_{3}} \oplus \cdots=\bigoplus_{i=1}^{\infty} T_{i}, \\
& X_{\infty}=X \oplus X \oplus X \oplus \cdots=\bigoplus_{i=1}^{\infty} X_{i} .
\end{aligned}
$$

Fix $f=\bigoplus_{i=1}^{\infty} c_{i} K\left(\lambda_{i}, \cdot\right) \xi_{i}$ satisfying

$$
\sum_{i=1}^{\infty}\left|c_{i}\right|^{2}\left\|K\left(\lambda_{i}, \cdot\right) \xi_{i}\right\|^{2}<\infty
$$

where $c_{i} \neq 0$ for all $i$. Thus $f \in \mathscr{K}_{\infty}^{\prime}$. Define

$$
=\operatorname{cl}\left\{\bigoplus_{i=1}^{\infty} p(T) c_{i} K\left(\lambda_{i}, \cdot\right) \xi_{i}: p \text { is a polynomial }\right\} .
$$

Since $f \in \mathscr{M}, \mathscr{M} \neq \emptyset$. Now clearly $\mathscr{M}$ is closed subspace of $\mathscr{K}_{\infty}^{\prime}$ and we have

$$
T_{\infty} f=\bigoplus_{i=1}^{\infty} c_{i} T K\left(\lambda_{i}, \cdot\right) \xi_{i}=\bigoplus_{i=1}^{\infty} c_{i} \lambda_{i} K\left(\lambda_{i}, \cdot\right) \xi_{i} .
$$

Thus $T_{\infty} f \in \mathscr{M}$ and so $\mathscr{M} \in \operatorname{Lat}\left(T_{\infty}\right)$. But $\operatorname{Lat}(T) \subseteq$ $\operatorname{Lat}(X)$;; thus $\operatorname{Lat}\left(T_{\infty}\right) \subseteq \operatorname{Lat}\left(X_{\infty}\right)$ and we get $\mathscr{M} \in \operatorname{Lat}\left(X_{\infty}\right)$. Therefore $X_{\infty} f \in \mathscr{M}$ and so there exists a sequence $\left\{p_{n}\right\}_{n}$ of polynomials such that

$$
\begin{aligned}
\oplus_{i} c_{i} p_{n}(T) c_{i} K\left(\lambda_{i}, \cdot\right) \xi_{i} & \longrightarrow \\
X_{\infty} f & =\oplus_{i} c_{i} \psi(T) c_{i} K\left(\lambda_{i}, \cdot\right) \xi_{i}
\end{aligned}
$$

in $\mathscr{K}_{\infty}^{\prime}$. Thus $\oplus_{i} c_{i}\left(p_{n}\left(\lambda_{i}\right)-\psi\left(\lambda_{i}\right)\right) K\left(\lambda_{i}, \cdot\right) \xi_{i} \rightarrow 0$ in $\mathscr{K}_{\infty}^{\prime}$ and since for all $i$

$$
\begin{aligned}
& \left\|\oplus_{i} c_{i}\left(p_{n}\left(\lambda_{i}\right)-\psi\left(\lambda_{i}\right)\right) K\left(\lambda_{i}, \cdot\right) \xi_{i}\right\| \\
& \quad \geq\left\|c_{i}\left(p_{n}\left(\lambda_{i}\right)-\psi\left(\lambda_{i}\right)\right) K\left(\lambda_{i}, \cdot\right) \xi_{i}\right\|,
\end{aligned}
$$

we get $\sup _{i}\left\|c_{i}\left(p_{n}\left(\lambda_{i}\right)-\psi\left(\lambda_{i}\right)\right) K\left(\lambda_{i}, \cdot\right) \xi_{i}\right\| \rightarrow 0$ as $n \rightarrow \infty$. But

$$
\begin{aligned}
\| c_{i} & \left(p_{n}\left(\lambda_{i}\right)-\psi\left(\lambda_{i}\right)\right) K\left(\lambda_{i}, \cdot\right) \xi_{i} \|^{2} \\
& =\left|c_{i}\left(p_{n}\left(\lambda_{i}\right)-\psi\left(\lambda_{i}\right)\right)\right|^{2}\left\langle K\left(\lambda_{i}, \lambda_{i}\right) \xi_{i}, \xi_{i}\right\rangle \\
& =\left|c_{i}\right|^{2}\left|p_{n}\left(\lambda_{i}\right)-\psi\left(\lambda_{i}\right)\right|^{2}\left\|K\left(\lambda_{i}, \lambda_{i}\right)^{1 / 2} \xi_{i}\right\|^{2}
\end{aligned}
$$

and $K\left(\lambda_{i}, \lambda_{i}\right)$ is invertible; thus for all $i,\left|\left(p_{n}-\psi\right)\left(\lambda_{i}\right)\right| \rightarrow 0$ as $n \rightarrow \infty$. This implies that $\left(p_{n}(T)-X\right) g \rightarrow 0$ for all $g$ in the finite linear combinations of

$$
Y=\left\{K\left(\lambda_{i}, \cdot\right) \xi: i \in \mathbb{N}, \xi \in \mathbb{C}^{n}\right\}
$$

that is a total subset of $\mathscr{K}$. At this time the proof is complete.

Let $\psi,\left\{p_{n}\right\}_{n}$, and $F=\left\{\lambda_{i}\right\}_{i}$ be defined as in the proof of Theorem 12. At the end of the proof of Theorem 12, we saw that, for all $i,\left|\left(p_{n}-\psi\right)\left(\lambda_{i}\right)\right| \rightarrow 0$ as $n \rightarrow \infty$. Now we ask the following question.

Question 13. In the proof of Theorem 12, is it true that $\sup _{i}\left|\left(p_{n}-\psi\right)\left(\lambda_{i}\right)\right| \rightarrow 0$ as $n \rightarrow \infty$ ?

If the answer of Question 13 is positive, then $\left\|p_{n}\right\|_{F} \leq M$ for some $M>0$ and we may have the following corollary. Note that the special case of this corollary has been proved as Theorem 2 in [4], only whenever $\Omega$ is a Carathéodory region.

Corollary 14. If $T$ is in $B_{n}(\Omega)$ where $\Omega \subset \mathbb{C}$ is a domain such that $\sigma(T)=\bar{\Omega}$ is a spectral set for $T$, then $T$ is reflexive. 
Proof. Let $K$ be a g.B.k. on $\Omega$ and let $X \in \operatorname{AlgLat}(T)$. By Theorem 12 , there exists a sequence of polynomials $\left\{p_{n}\right\}_{n}$ such that $p_{n}(T)$ converges in the finite linear combinations of

$$
\left\{K(\lambda, \cdot) \xi: \lambda \in F, \xi \in \mathbb{C}^{n}\right\}
$$

that is a total subset of $\mathscr{K}$, where $F=\left\{\lambda_{n}\right\}_{n=1}^{\infty}$ was a dense set in $\Omega$. Also, $\sup _{i}\left|\left(p_{n}-\psi\right)\left(\lambda_{i}\right)\right| \rightarrow 0$ as $n \rightarrow \infty$. This implies that $\left\|p_{n}\right\|_{\Omega}=\left\|p_{n}\right\|_{F} \leq M$ for some $M>0$. Now since $\sigma(T)=\bar{\Omega}$ is a spectral set for $T$, we conclude that $\left\|p_{n}(T)\right\| \leq M$. Since the unit ball of $B(\mathscr{K})$ is compact in the weak operator topology, by passing to a subsequence if necessary, we may assume that $p_{n}(T) \rightarrow A$ in the weak operator topology. Therefore, $p_{n}(T) K(\lambda, \cdot) \xi \rightarrow A K(\lambda, \cdot) \xi$ weakly. But

$$
p_{n}(T) K(\lambda, \cdot) \xi=p_{n}(\lambda) K(\lambda, \cdot) \xi \longrightarrow \psi(\lambda) K(\lambda, \cdot) \xi .
$$

Hence, $A K(\lambda, \cdot) \xi=\psi(\lambda) K(\lambda, \cdot) \xi$, where, by the proof of Theorem $12, \psi$ is a function in $H^{\infty}(\Omega)$ and satisfies $X K(\lambda, \cdot)=$ $\psi(\lambda) K(\lambda, \cdot)$ for all $\lambda$ in $\Omega$. From this we conclude that $A=$ $X$, so $X \in W(T)$. Therefore, $\operatorname{AlgLat}(T) \subset W(T)$ and $T$ is reflexive. This completes the proof.

\section{Conflicts of Interest}

The authors declare that there are no conflicts of interest regarding the publication of this paper.

\section{References}

[1] M. J. Cowen and R. G. Douglas, "Complex geometry and operator theory," Acta Mathematica, vol. 141, no. 3-4, pp. 187261, 1978.

[2] R. E. Curto and N. Salinas, "Generalized Bergman kernels and the Cowen-Douglas theory," American Journal of Mathematics, vol. 106, no. 2, pp. 447-488, 1984.

[3] S. Richter, "Invariant subspaces in Banach spaces of analytic functions," Transactions of the American Mathematical Society, vol. 304, no. 2, pp. 585-616, 1987.

[4] K. Seddighi and B. Yousefi, "On the reflexivity of operators on function spaces," Proceedings of the American Mathematical Society, vol. 116, no. 1, pp. 45-52, 1992.

[5] B. Yousefi and S. Foroutan, "On the multiplication operators on spaces of analytic functions," Studia Mathematica, vol. 168, no. 2, pp. 187-191, 2005.

[6] K. Zhu, "Operators in Cowen-Douglas classes," Illinois Journal of Mathematics, vol. 44, no. 4, pp. 767-783, 2000.

[7] K. Zhu, "Irreducible multiplication operators on spaces of analytic functions," The Journal of Operator Theory, vol. 51, no. 2, pp. 377-385, 2004.

[8] A. L. Shields and L. J. Wallen, "The commutants of certain Hilbert space operators," Indiana University Mathematics Journal, vol. 20, pp. 777-788, 1970/1971.

[9] K. Seddighi, Von Neumann operators in $\mathrm{B}_{1}(\Omega)$, University of Indiana, 1981.

[10] L. Bagheri and B. Yousefi, "Reflexivity of the shift operator on some BK spaces," Rendiconti del Circolo Matematico di Palermo Serie II, vol. 63, no. 1, pp. 91-96, 2014.

[11] L. Chen, "On intertwining operators via reproducing kernels," Linear Algebra and its Applications, vol. 438, no. 9, pp. 36613666, 2013.
[12] L. Chen, R. G. Douglas, and K. Guo, "On the double commutant of Cowen-Douglas operators," Journal of Functional Analysis, vol. 260, no. 7, pp. 1925-1943, 2011.

[13] K.-y. Guo and H.-s. Huang, "Reducing subspaces of multiplication operators on function spaces," Applied Mathematics-A Journal of Chinese Universities Series B, vol. 28, no. 4, pp. 395404, 2013.

[14] K. Ji, C. Jiang, D. K. Keshari, and G. Misra, "Flag structure for operators in the Cowen-Douglas class," Comptes Rendus Mathematique, vol. 352, no. 6, pp. 511-514, 2014.

[15] A. Koranyi and G. Misra, "A classification of homogeneous operators in the Cowen-Douglas class," Advances in Mathematics, vol. 226, no. 6, pp. 5338-5380, 2011.

[16] B. Yousefi and S. Khoshdel, "Reflexivity of Powers of the Multiplication Operator on Special Function Spaces," Acta Mathematica Scientia, vol. 32, no. 6, pp. 2279-2284, 2012.

[17] J. B. Conway, The Theory of Subnormal Operators, vol. 36 of Mathematical Surveys and Monographs, American Mathematical Society, Providence, RI, USA, 1991. 


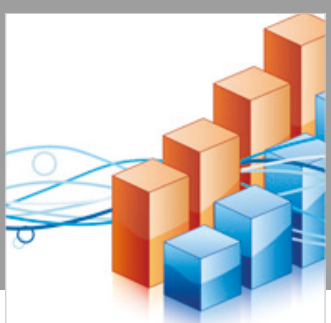

Advances in

Operations Research

\section{-n-m}
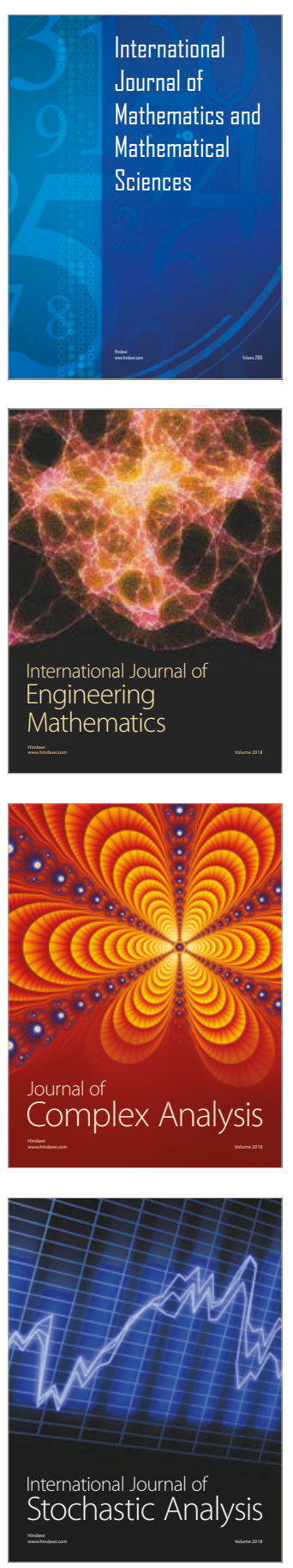
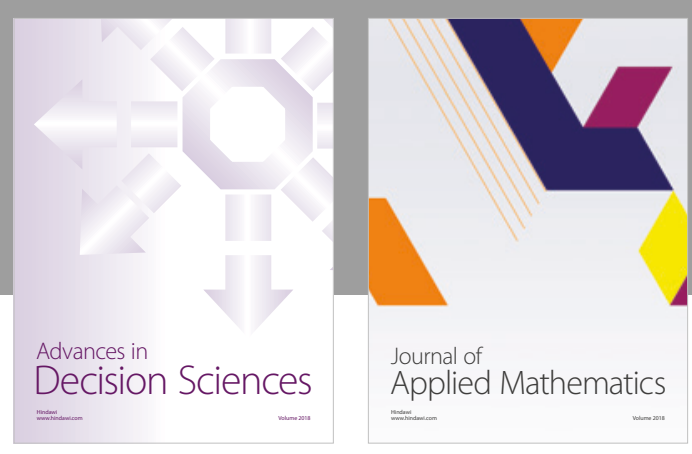

Journal of

Applied Mathematics
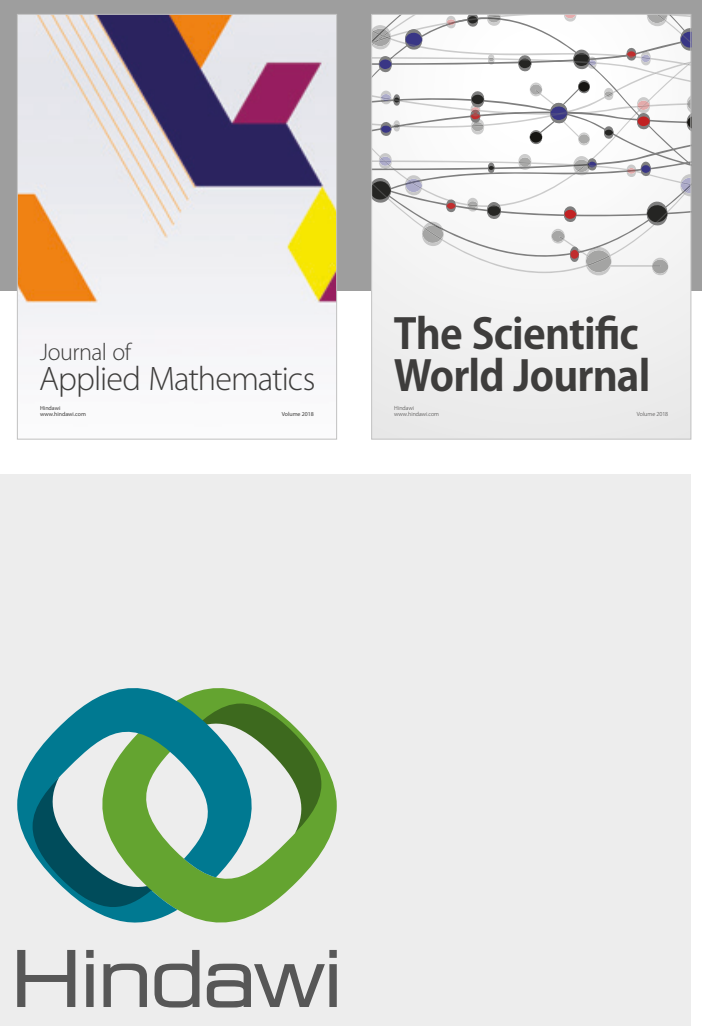

Submit your manuscripts at

www.hindawi.com

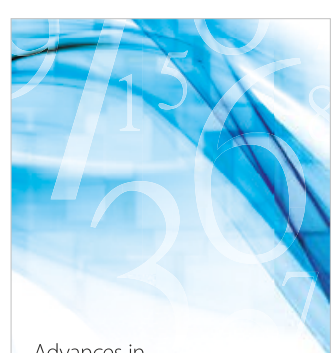

Advances in
Numerical Analysis
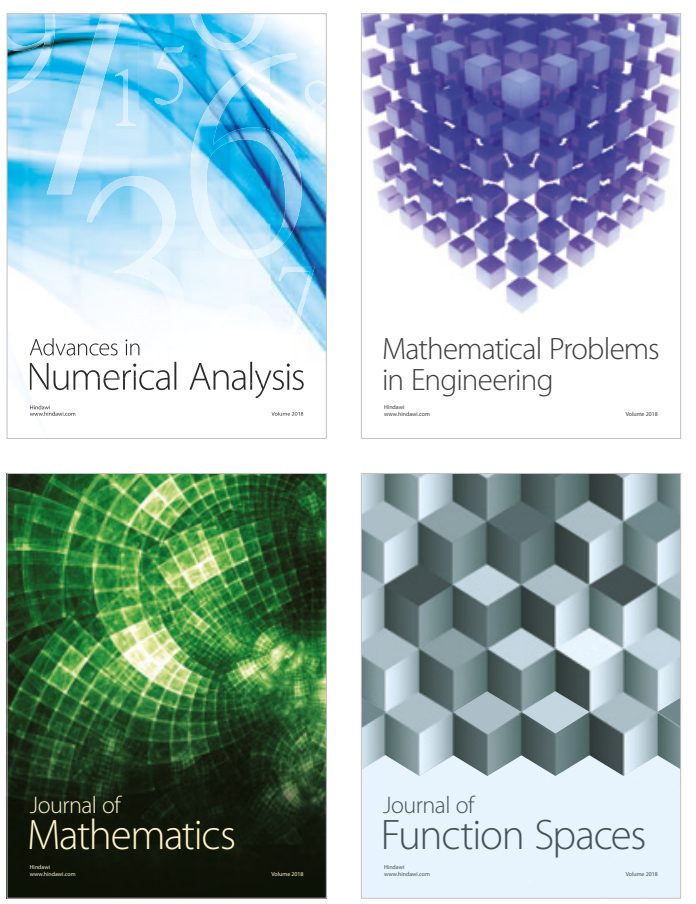

Mathematical Problems in Engineering

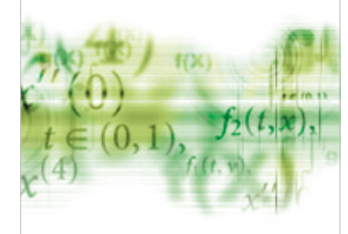

International Journal of

Differential Equations

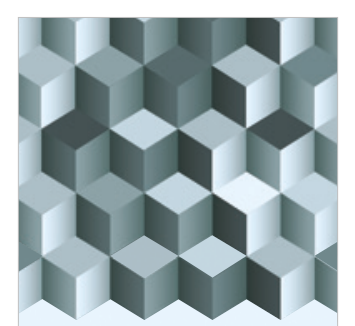

Journal of

Function Spaces

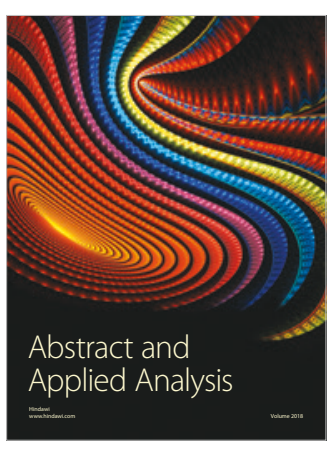

The Scientific

World Journal

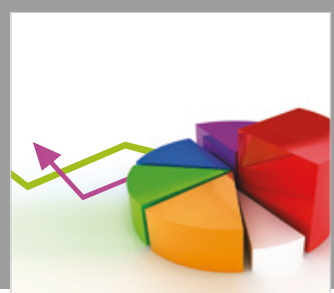

Journal of

Probability and Statistics
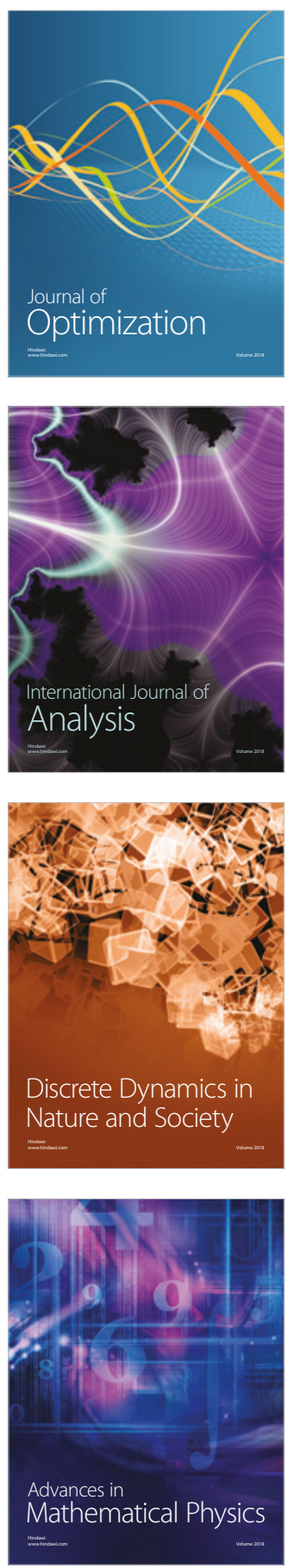